\section{Platelet Normalized Serum Vascular Endothelial Growth Factor Levels in Progressive Pediatric Solid Malignancies}

We read with interest the recently published article by Pramanik, et al. [1]. The study reported inconsistent trends in serum vascular endothelial growth factor (VEGF) and thrombospondin-1 (TSP1) in 108 patients with progressive pediatric solid tumors who received metronomic chemotherapy or placebo [1]. While higher baseline serum VEGF levels predicted inferior overall survival, authors found that responders with metronomic chemotherapy had significantly lower VEGF levels at baseline compared with non-responders. Further, there was no association of serial VEGF levels with response to metronomic chemotherapy.

Of note, VEGF is released from the $\alpha$ granules on platelet activation during sample collection and therefore, serum levels are considered as an inaccurate indicator of actual measurement of circulating VEGF [2]. Patients with disseminated cancer may have a higher platelet count and carry even higher VEGF per platelets compared with general population [3]. Thus, plasma is preferred over serum to measure VEGF because collecting blood in citrate tubes avoids platelet activation and therefore preventing the spurious high VEGF levels released from platelets [4]. Since the authors used serum to measure VEGF levels in pediatric patients with solid tumors in the study, the results must be interpreted with caution [1]. However, serum VEGF levels normalized to patient's platelet count provides serum VEGF/ platelet, which can neutralize the effect of VEGF released from platelets while withdrawing blood [5]. Therefore, the authors may consider analyzing the data after calculating serum VEGF/platelet for all measurements in individual patients, if data on platelet count is available. It will be interesting to see if a consistent pattern is then noticed between serum VEGF/platelet with the response to metronomic chemotherapy and survival outcomes.

Further, the authors described the effect of baseline serum VEGF levels with overall survival in overall population as well as responders in patients randomized to metronomic chemotherapy arm [1]. While this finding is interesting, it is an exploratory subgroup finding in a small number of patients, which can be interpreted as hypothesis generating at best and therefore, should be interpreted with utmost caution.

Atul Batra Department of Medical Oncology, Tom Baker Cancer Center; and University of Calgary, Calgary, Alberta, Canada. batraatul85@gmail.com

\section{REFERENCES}

1. Pramanik R, Tyagi A, Agarwala S, Vishnubhatla S, Dhawan D, Bakhshi S. Evaluation of vascular endothelial growth factor (VEGF) and Thrombospondin-1 as biomarkers of metronomic chemotherapy in progressive pediatric solid malignancies. Indian Pediatr. 2020;57: 508-11.

2. Webb NJ, Bottomley MJ, Watson CJ, Brenchley PE. Vascular endothelial growth factor (VEGF) is released from platelets during blood clotting: Implications for measurement of circulating VEGF levels in clinical disease. Clin Sci. 1998;94:395-404.

3. Salven P, Orpana A, Joensuu H. Leukocytes and platelets of patients with cancer contain high levels of vascular endothelial growth factor. Clin Cancer Res. 1999;5: $\quad$ 487-91.

4. Banks RE, Forbes MA, Kinsey SE, et al. Release of the angiogenic cytokine vascular endothelial growth factor (VEGF) from platelets: Significance for VEGF measurements and cancer biology. Br J Cancer. 1998;77:956-64.

5. George ML, Eccles SA, Tutton MG, Abulafi AM, Swift RI. Correlation of plasma and serum vascular endothelial growth factor levels with platelet count in colorectal cancer: Clinical evidence of platelet scavenging? Clin Cancer Res. 2000;6:3147-52.

\section{AUTHORS'REPLY}

We appreciate the comments and suggestions by the reader. Available literature shows that there is a controversy regarding the best blood compartment and the best test to measure VEGF in cancer patients. VEGF in cancer patients is the sum total of platelet derived VEGF as well as other sources like neoangiogenesis in the tumor tissue. One of the studies showed that the best discrimination between healthy volunteers and cancer patients was observed in platelet poor plasma (PPP). As generating plasma induces platelet activation with consequent VEGF release from platelets, citrate-theophylline-adenosinedipyridamole plasma was suggested by some authors to evaluate VEGF [1]. Serum VEGF is more practical because VEGF levels in citrated plasma are low and lie close to the limits of ELISA sensitivity. Some studies have shown that a standardized measurement of serum VEGF, normalized by the patient's platelet count, which gives a value of serum VEGF per platelet, can be a useful parameter [2].

We had our baseline platelet counts for all the patients but the corresponding platelet counts for subsequent follow up (A2 and A3) assessments were not available for all patients [3]. Hence, we restricted our analysis to baseline values only. On applying pair wise correlation to the baseline platelet count and serum VEGF, we found an insignificant correlation; $\mathrm{r}=0.16(P=0.09)$ (Fig. 1a). Baseline serum VEGF showed a significant positive correlation with baseline VEGF per platelet $(\mathrm{r}=0.81, P<0.0001)$ (Fig. 1b). As the serum VEGF and VEGF/per platelet correlate significantly, both are likely to follow similar trends; this implies that we are likely to have similar observations, whether we use serum VEGF or VEGF/platelet.

Similar observations were reported by Vermeulen, et al. [4]; they commented that in view of the lack of a strong association 

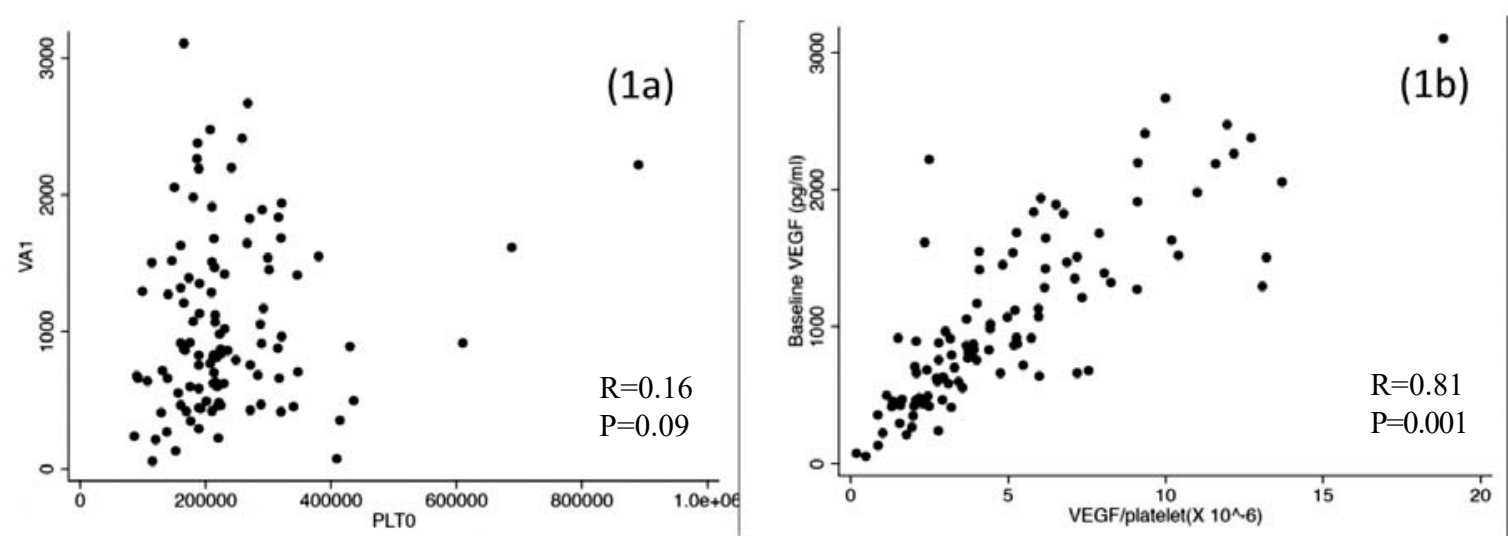

Fig. 1 Scatter plots showing correlation between (a) baseline VEGF and baseline platelet counts, and (b) baseline VEGF and baseline VEGF per platelet.

between serum VEGF and platelet count, and the association of serum VEGF with the degree of stimulation of endothelial cell proliferation in vitro, measuring serum VEGF might be more suitable in cancer patients than measuring plasma VEGF. Also, it has been postulated that at least part of the VEGF in platelets represents that endocytosed from the plasma due to their scavenging effect [4]. So, measuring the entire collection (serum VEGF) may not be less appropriate.

Further, most previous studies on metronomic chemotherapy had measured serum VEGF and we intended to be consistent and comparable to them $[5,6]$. Hence, our conclusion remains the same that VEGF is not a reliable biomarker for metronomic chemotherapy, but the best test for VEGF still remains an illusion.

\section{REFERENCES}

Raja Pramanik and Sameer BaKhshi* Department of Medical Oncology, All India Institute of Medical Sciences, New Delhi, India. *sambakh@hotmail.com

1. Wynendaele W, Derua R, Hoylaerts MF, et al. Vascular endothelial growth factor measured in platelet poor plasma allows optimal separation between cancer patients and volunteers: A key to study an angiogenic marker in vivo? Ann Oncol. 1999;10:965-71.

2. George ML, Eccles SA, Tutton MG, Abulafi AM, Swift RI. Correlation of plasma and serum vascular endothelial growth factor levels with platelet count in colorectal cancer: Clinical evidence of platelet scavenging? Clin Cancer Res. 2000;6:3147-152.

3. Pramanik R, Tyagi A, Agarwala S, Vishnubhatla S, Dhawan D, Bakhshi S. Evaluation of vascular endothelial growth factor (VEGF) and thrombospondin-1 as bio-markers of metronomic chemotherapy in progressive pediatric solid malignancies. Indian Pediatr. 2020; 57:508-11.

4. Vermeulen PB, Salven P, Benoy I, Gasparini G, Dirix LY. Blood platelets and serum VEGF in cancer patients. Br J Cancer. 1999;79:370-73.

5. Kesari S, Schiff D, Doherty L, et al. Phase II study of metronomic chemotherapy for recurrent malignant gliomas in adults. Neuro Oncol. 2007;9:354-63.

6. Kieran MW, Turner CD, Rubin JB, et al. A feasibility trial of antiangiogenic (metronomic) chemotherapy in pediatric patients with recurrent or progressive cancer. J Pediatr Hematol Oncol. 2005;27;573-81.

\section{Psychosocial Wellness During the COVID- 19 Pandemic: Building an ARCH}

The COVID-19 pandemic is associated with significant morbidity and mortality. However, little attention has been devoted to psychological factors, emotional distress and social disruption in children. It is believed that the disease, multiplied by forced quarantine and nationwide lockdowns can induce acute panic, anxiety, obsessive behaviors, paranoia, depression and post-traumatic stress disorder (PTSD) [1]. The pandemic is likely to be followed by a 'second pandemic' of mental health crises [2]. This necessitates a comprehensive public health response with innovations for providing mental health care, while maintaining social distancing.

To support and protect psychosocial well-being of children, we propose the ARCH model for mental health workers, parents and teachers. ARCH is an acronym for Adapt and attempt, Resilience, Collaboration and care, and Humor and humility.

In an uncertain and evolving situation, children may be encouraged to adapt to the current scenario and attempt solutions in a new normal, rather than wait for familiar comfort zones. Options for physical activities have been drastically reduced. Children tend to spend their excessive free time on television or mobile phone [3]. Introducing positive adaptation skills is essential. Children need to feel safe, secure, and positive about 